\title{
Simple pathological examination technique for detection of cancer located at the surgical margin of the stomach
}

\author{
Kotaro Okuda $\cdot$ Sho Ishihara $\cdot$ Yasuko Fujita $\cdot$ \\ Noriko Yamamoto $\cdot$ Mitsuo Kishimoto • \\ Eiichi Konishi • Yo Kato $\cdot$ Akio Yanagisawa
}

Received: 25 August 2012/ Accepted: 21 April 2013/Published online: 17 May 2013

(C) The International Gastric Cancer Association and The Japanese Gastric Cancer Association 2013

\begin{abstract}
Background The technique for examining surgical resection margins described in the Japanese Classification of Gastric Carcinoma is based on the examination of continuous infiltration by the primary tumor, and discontinuous lesions such as multiple cancers are not examined. However, examining lesions-particularly cancers-at the resection margins is important for the prevention of cancers in the remaining stomach that result from cancer remnants (remnant gastric cancer).

Methods The clinical usefulness of a simple pathological examination technique for detecting cancer located at the surgical margin of the stomach was studied. A specimen 5-8 $\mathrm{mm}$ wide was resected from the surgical cut margin along the entire circumference of the stomach. When the pathological margin was positive for cancer, the surgical margin was also examined, and cases that were positive for cancer were regarded as marginally positive.
\end{abstract}

K. Okuda $(\bowtie) \cdot$ Y. Fujita · M. Kishimoto · E. Konishi ·

A. Yanagisawa

Department of Surgical Pathology, Kyoto Prefectural University

of Medicine, 465 Kajiicho, Kamigyou-ku,

Kyoto 602-8566, Japan

e-mail: tarojibe@koto.kpu-m.ac.jp

K. Okuda

Department of Gastroenterology, Kyoto Kujo Hospital,

10 Karahashi-rajyomon-cho, Minami-ku, Kyoto 601-8453, Japan

S. Ishihara

Ishihara Clinic, 1-2-10 Hamazaki, Asaka,

Saitama 351-0033, Japan

N. Yamamoto $\cdot$ Y. Kato

Division of Pathology, The Cancer Institute of the Japanese

Foundation for Cancer Research, 3-8-31 Ariake,

Koto-ku, Tokyo 135-8550, Japan
Results Of the 1,498 patients with early gastric cancer who were examined using this method, $17(1.1 \%)$ were marginally positive for multiple cancers, and 8 of these 17 patients $(57 \%)$ had microcancers $<5 \mathrm{~mm}$ in diameter.

Conclusion This method is simple and useful for detecting cancer involving the surgical margin, which occurs at a rate of $1.1 \%$, making it possible to prevent remnant gastric cancer by reoperation.

Keywords Early gastric cancer - Residual cancer . Second primary cancers $\cdot$ Surgery

\section{Introduction}

The technique for examining surgical resection margins described in the Japanese Classification of Gastric Carcinoma involves preparing a specimen by making as many incisions as required parallel to the lesser curvature line and examining the specimen [1]. This technique is based on the examination of continuous infiltration by the primary tumor, and discontinuous lesions such as multiple cancers are not examined. However, examining lesionsparticularly cancers-at the resection margins is important for the prevention of cancers in the remaining stomach that result from cancer remnants (remnant gastric cancer). Thus, surgical resection margins along the entire circumference of the stomach should be examined in all patients. However, the examination method specified in the guideline is inefficient because it requires the resection of a large number of specimens. Since 1987, we have identified and diagnosed cancers at the surgical resection margins in patients who have undergone gastric resection, excluding those who underwent total gastrectomy (mainly patients with early gastric cancer), using a technique that is unique 
to our pathological department. We have named this technique "the simple pathological examination technique for the surgical margin of the stomach." This report describes our evaluation of the clinical usefulness of this method.

\section{Patients and methods}

The subjects included 1,498 patients with early gastric cancer who underwent resection $(1,196$, distal gastrectomy; 153, proximal gastrectomy; 113, segmental gastrectomy; 36, pylorus-preserving gastrectomy) at the Cancer Institute Hospital Department of Surgery between 1988 and 1997, or at the University Hospital Department of Digestive Surgery, Kyoto Prefectural University of Medicine, between 2003 and 2011. The resection margins of these patients were examined using the simple pathological examination technique. First, a region 5-8 $\mathrm{mm}$ wide was resected from the surgical margin along the entire circumference of the stomach to obtain a specimen of the pathological margin (Fig. 1). When the pathological margin was positive for cancer, the counterpart surface (surgical margin) was resected to obtain a specimen. When the surgical margin was positive for cancer, the lesion was diagnosed as marginally positive. We did not obtain specimens of the surgical margins from patients at early stages because there is a possibility that the electrosurgical knife may damage the surgical resection margin. When a stapled anastomosis was performed, we did not obtain specimens. The Japanese Classification of Gastric Carcinoma was used to classify the endoscopic appearance and determine the histological classification of the lesions [1]. We performed a clinicopathological examination of the primary tumors and lesions at the resection margins in patients who were diagnosed with marginally positive lesions using the foregoing method and evaluated the usefulness of this method.

\section{Results}

Clinicopathological findings in patients with marginally positive lesions

Of the 1,498 subjects, $17(1.1 \%)$ were diagnosed with marginally positive lesions: $12(1.0 \%)$ were diagnosed by distal gastrectomy, $2(1.3 \%)$ were diagnosed by proximal gastrectomy, and $3(2.7 \%)$ were diagnosed by segmental resection. Fifteen subjects had double cancer, and 1 subject each had triple and quintuple cancer. All patients had marginally positive lesions because of a secondary focus (lesion at the resection margin). The clinicopathological findings of the primary and secondary foci in the 17 marginally positive

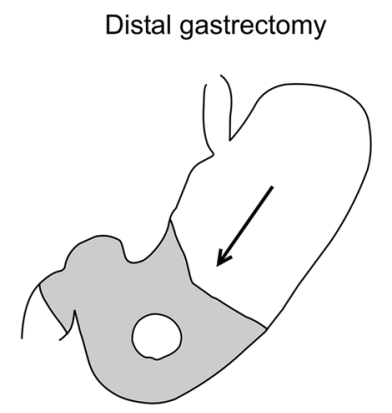

\section{Proximal gastrectomy}
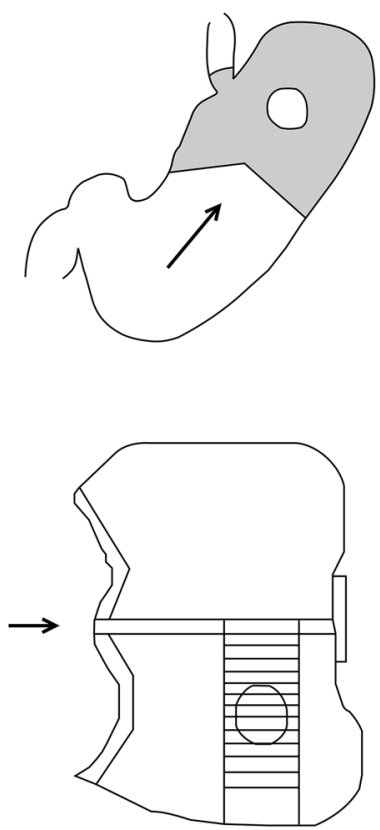

Segmental gastrectomy
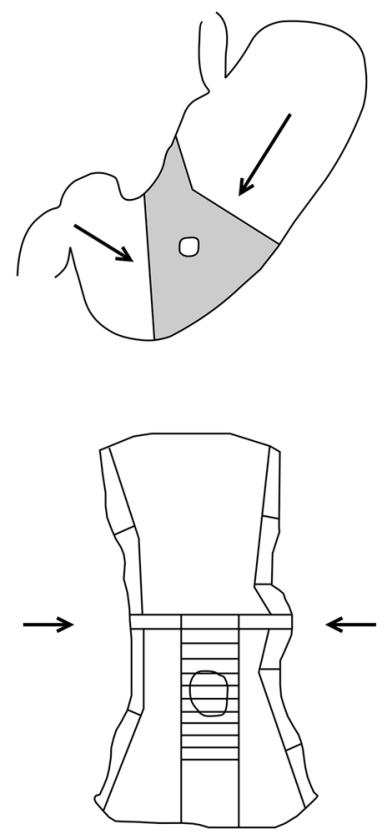

Fig. 1 The simple pathological examination technique is illustrated. Specimens, 5-8 $\mathrm{mm}$ wide, were resected from the surgical cut margin along the entire circumference of the stomach depending on each surgical procedure (arrow). When the pathological margin was positive for cancer, the surgical cut margin was also examined 
Table 1 Clinicopathological findings of the cases diagnosed as marginally positive for cancer

\begin{tabular}{|c|c|c|c|c|c|c|c|c|}
\hline \multirow[t]{2}{*}{ Case [age (years), sex] } & \multicolumn{4}{|c|}{ Primary cancer } & \multicolumn{4}{|c|}{ Cancer located at the pathological margin } \\
\hline & Histology & Macroscopic type & Size $(\mathrm{mm})$ & Depth & Histology & Macroscopic type & Size $(\mathrm{mm})$ & Depth \\
\hline 1. $67, \mathrm{M}^{\mathrm{a}}$ & tub1 & IIc & 40 & $\operatorname{sm} 2$ & tub1 & IIc & 13 & $\mathrm{~m}$ \\
\hline 2. $53, \mathrm{M}$ & tub1 & IIc & 10 & $\operatorname{sm} 2$ & tub1 & $\mathrm{IIb}$ & 8 & $\mathrm{~m}$ \\
\hline 3. $74, \mathrm{M}$ & tub1 & IIc & 18 & $\mathrm{~m}$ & tub1 & $\mathrm{IIb}$ & 5 & $\mathrm{~m}$ \\
\hline 4. $40, \mathrm{M}$ & tub1 & $\mathrm{IIa}+\mathrm{IIc}$ & 20 & $\mathrm{~m}$ & tub1 & IIc & 2 & $\mathrm{~m}$ \\
\hline 5. $47, \mathrm{M}$ & tub1 & IIa & 60 & $\mathrm{sm} 2$ & tub2 & IIc & 20 & $\operatorname{sm} 2$ \\
\hline 6. $61, \mathrm{M}$ & tub1 & IIc & 20 & $\mathrm{sm} 2$ & tub1 & $\mathrm{IIb}$ & 4 & $\mathrm{~m}$ \\
\hline 7. $63, \mathrm{M}$ & tub1 & IIc & 15 & $\mathrm{~m}$ & tub1 & IIa & 5 & $\mathrm{~m}$ \\
\hline 8. $66, \mathrm{M}$ & tub1 & IIc & 21 & $\mathrm{~m}$ & tub2 & IIc & 3 & $\mathrm{~m}$ \\
\hline $9.75, \mathrm{M}$ & tub1 & IIa & 40 & $\mathrm{~m}$ & sig & $\mathrm{IIb}$ & 50 & $\mathrm{~m}$ \\
\hline 10. $70, \mathrm{M}^{\mathrm{a}}$ & tub1 & IIa & 43 & $\mathrm{~m}$ & por2 & $\mathrm{IIb}$ & 7 & $\mathrm{~m}$ \\
\hline 11. $64, \mathrm{M}$ & tub1 & IIc & 5 & $\mathrm{~m}$ & sig & IIc & 2 & $\mathrm{~m}$ \\
\hline 12. $53, \mathrm{M}^{\mathrm{a}}$ & tub1 & IIc & 52 & $\mathrm{sm} 2$ & sig & $\mathrm{IIb}$ & 5 & $\mathrm{sm} 2$ \\
\hline 13. $58, \mathrm{M}$ & por2 & IIc & 21 & $\mathrm{sm} 2$ & tub2 & IIc & 7 & $\mathrm{sm} 2$ \\
\hline 14. 59, F & sig & IIc & 18 & $\mathrm{sm} 2$ & sig & IIc & 5 & $\mathrm{~m}$ \\
\hline 15. $82, \mathrm{M}^{\mathrm{a}}$ & tub1 & $\mathrm{IIa}+\mathrm{IIb}$ & 30 & $\mathrm{~m}$ & tub1 & $\mathrm{IIb}$ & 12 & $\mathrm{~m}$ \\
\hline \multirow[t]{2}{*}{ 16. $60, \mathrm{M}$} & sig & IIa + IIc & 16 & $\operatorname{sm} 2$ & tub2 & IIb & 13 & $\mathrm{~m}$ \\
\hline & & & & & tub2 & IIb & 10 & $\mathrm{~m}$ \\
\hline \multirow[t]{4}{*}{ 17. $71, \mathrm{~F}^{\mathrm{a}}$} & tub1 & I & 35 & $\mathrm{~m}$ & tub1 & IIa & 7 & $\mathrm{~m}$ \\
\hline & & & & & tub1 & $\mathrm{IIb}$ & 4 & $\mathrm{~m}$ \\
\hline & & & & & tub1 & IIa & 6 & $\mathrm{~m}$ \\
\hline & & & & & tub1 & $\mathrm{IIb}$ & 1 & $\mathrm{~m}$ \\
\hline
\end{tabular}

$m$ mucosa, $s m 1$ penetration of submucosal layer less than $500 \mu \mathrm{m}$ from muscularis mucosa, $s m 2$ penetration of $500 \mu \mathrm{m}$ or more, $t u b 1$ welldifferentiated tubular adenocarcinoma; $t u b 2$ moderately differentiated tubular adenocarcinoma, por 2 poorly differentiated adenocarcinoma (nonsolid type), sig signet-ring cell carcinoma, I protruding, IIa superficial elevated, II $b$ superficial flat, IIc superficial depressed

${ }^{a}$ Re-resected case

Fig. 2 Photograph of cut specimen obtained from patient 15 (a) and its schema (b). Cancer cells were first observed on the pathological margin (arrow 1), and the opposite surgical margin was subsequently examined. The lesion was diagnosed as marginally positive on the basis of the detection of lesions (arrow 2) a

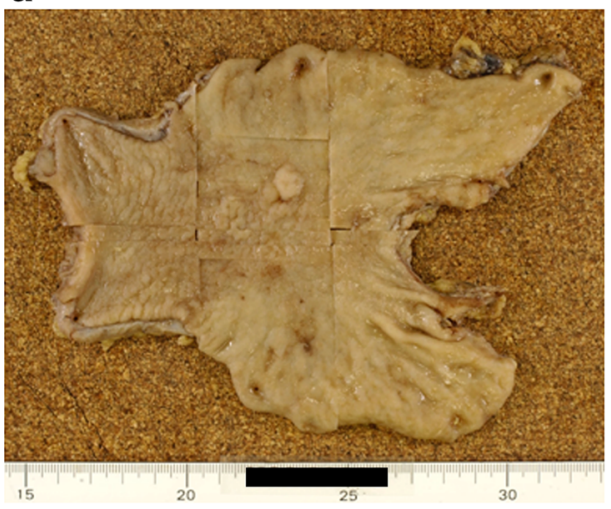

b

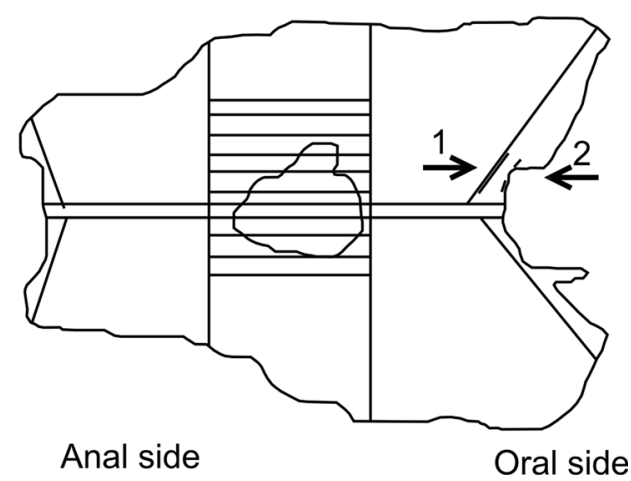

patients are shown in Table 1. Secondary foci, which were not diagnosed before or during the surgery, were diagnosed for the first time during the postoperative histopathological examination. At the secondary foci, 16 of 21 subjects $(76.2 \%)$ had a tumor with a maximum diameter of $10 \mathrm{~mm}$ or less, and 10 subjects $(47.6 \%)$ had microcancers measuring $5 \mathrm{~mm}$ or less in diameter. The histological types of 16 $(76.2 \%), 4(19 \%)$, and $1(4.8 \%)$ of the lesions were well- or moderately differentiated adenocarcinomas (tub1/tub2), signet-ring cell carcinomas (sig), and poorly differentiated adenocarcinoma (por), respectively, whereas 3 (14.3\%), 7 (33.3\%), and $11(52.4 \%)$ lesions were classified as superficial elevated (IIa), superficial depressed (IIc), and superficial flat (IIb), respectively. Submucosal invasion was evident in 3 patients $(14.3 \%)$, and mucosal invasion was evident in all other patients. 


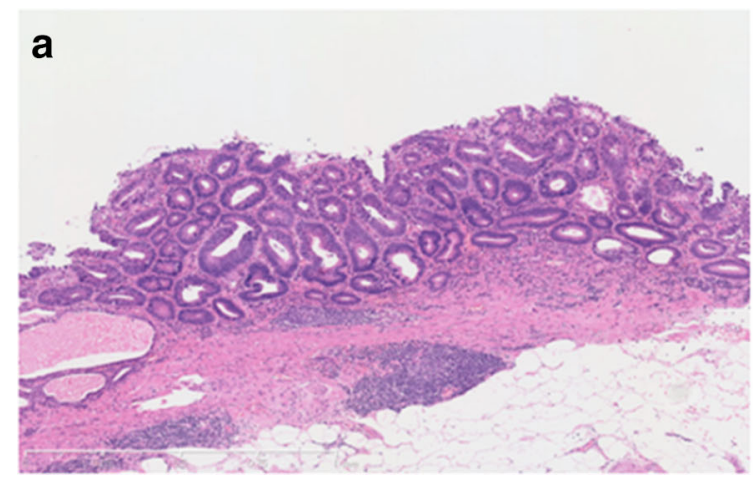

Fig. 3 a Histopathological findings at the margin on the oral side (pathological margin) in patient 15. Infiltration of well-differentiated adenocarcinoma (tub 1) was observed in the mucosal layers. b Histopathological findings at the margin on the oral side (surgical margin) in patient 15 . As in $\mathbf{a}$, infiltration of well-differentiated

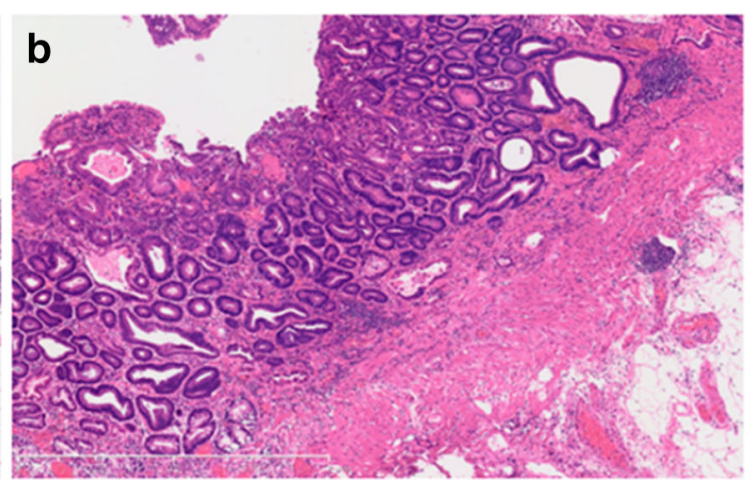

adenocarcinoma (tub 1) was observed in the mucosal layers, and the lesion was diagnosed as marginally positive. Partial ablation of the mucosal layer and injuries to the submucosal layer were artifacts resulting from the surgical margin
Fig. 4 Photograph of specimen resected from remnant stomach of patient 15 (a) and its schema (b). Residual cancer was observed on the anastomotic line (at arrow) a

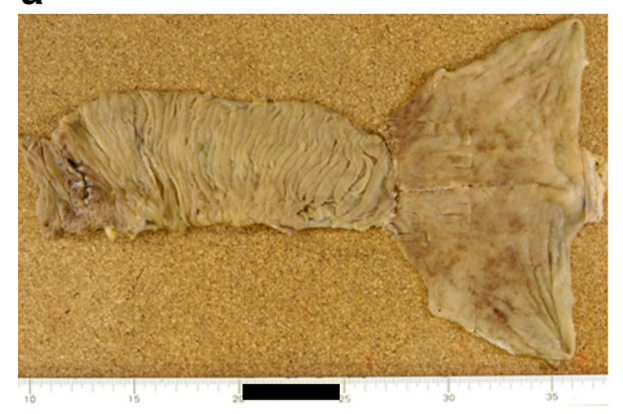

b

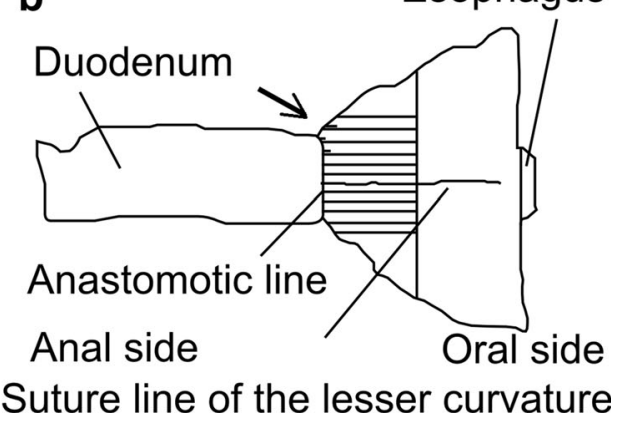

Relationship between the primary and secondary foci

The histological combinations of the primary and secondary foci were differentiated/differentiated in 10 patients $(58.8 \%)$, differentiated/undifferentiated or undifferentiated/differentiated in 6 patients $(35.3 \%)$, and undifferentiated/undifferentiated in 1 patient $(5.9 \%)$; the differentiated/differentiated combination was most often observed in patients with multiple cancers (Table 1). A photograph and schema of the resected specimens from patient 15 are shown in Fig. 2a, b; the pathological margin is shown in Fig. 3a, and a histopathological image of the surgical margin is shown in Fig. $3 b$.

\section{Evaluation of patients undergoing surgical re-resection}

Of the 17 patients with marginally positive lesions, 6 underwent re-resection of the remaining stomach, and of these, 5 patients $(83.3 \%)$ had remnant cancer in the remaining stomach. The other 11 patients refused surgery, underwent reoperation at other hospitals, or were outpatients with whom we lost contact. The photograph and schema of the resected specimen obtained during re-resection of the remaining stomach in patient 15 are

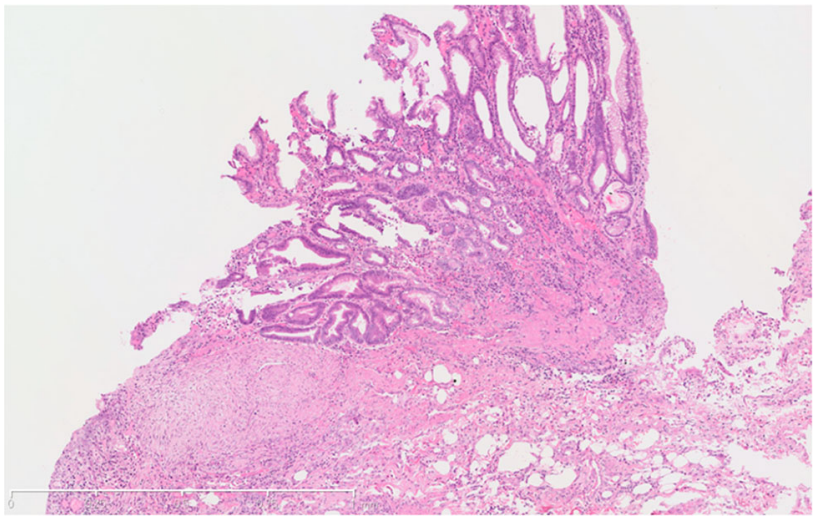

Fig. 5 Histopathological findings of specimen re-resected from the remnant stomach of patient 15 . Residual well-differentiated adenocarcinoma (tub 1) was observed in the mucosa on the anastomotic line

shown in Fig. 4a, b, and a histopathological image of the remnant gastric cancer is shown in Fig. 5.

Comparison between the two hospitals

Of the 1,208 subjects treated at the Cancer Institute Hospital Department of Surgery between 1988 and 1997, 14 
$(1.2 \%)$ were diagnosed with marginally positive lesions. Of the 290 subjects treated at the University Hospital Department of Surgery, Kyoto Prefectural University of Medicine, between 2003 and 2011, 3 (1.0\%) were diagnosed with marginally positive lesions. No significant difference was found between the two hospitals in this regard.

\section{Discussion}

The results of this study revealed that patients with early gastric cancer had marginally positive lesions resulting from the presence of multiple cancers rather than continuous infiltration of the primary cancer. Multiple cancers occur in patients with early gastric cancer at a frequency of 2.07-21\% [2-8], and multiple cancers are common in elderly patients with differentiated-type lesions $[3,5,9$, 10]. Further, multiple cancers often occur in the same background mucosa [2, 5]. The secondary foci of differentiated multiple cancers are distributed more widely than are those of undifferentiated types [9, 11]. Diagnostic examinations have indicated that secondary foci are distributed in segments located away from the main focus in many patients in whom secondary foci were overlooked, and many of the secondary foci were cancers in situ, microcancers with a maximum diameter of $5 \mathrm{~mm}$ or less, or type IIb cancers $[3,5,9,10,12]$.

Our examination indicated that 10 of 17 lesions at resection margins $(58.9 \%)$ were microcancers. Of all the completely resected gastric specimens, $8 \%$ had microcancers that were difficult to identify preoperatively, and the frequency of such cancers is said to increase further in patients with multiple gastric cancers and severe intestinal metaplasia [11]. Gastric resection lines can be extended to the favored sites of these multiple cancers; as the extent of resection decreases via reduction surgery, the possibility of extension increases [11]. In fact, in a previous report describing the examination of completely resected gastric specimens, secondary foci were accidentally found within $1 \mathrm{~cm}$ of the resection margin in 13 of 169 patients $(7.7 \%)$ with multiple cancers [13]. After subtotal gastrectomy, the incidence of remnant cancer was $1.7 \%$ in a European series [14], whereas the incidence was $1.1 \%$ in the current study and in a previous Japanese study [15]. The results of these studies reflect the clinicopathological characteristics of multiple cancers, suggesting that multiple cancers may be found at resection margins at a certain ratio when the resection margins of all patients are examined. Thus, we must consider that microgastric cancers are present at the surgical resection margins of early gastric cancers at a frequency of $1.1 \%$.

Previous studies have reported various approaches for the preoperative examination of such multiple microcancers; however, it is difficult to diagnose cancers that are undifferentiated, those less than $3 \mathrm{~mm}$ in diameter, or those which do not accompany ulcer scars, thus posing clinical limitations [16, 17]. The results obtained from two hospitals over two different time periods were not significantly different. Despite the development of improved endoscopic imaging technology, in the study of the two hospitals in which patients had similar positive margins, there is a limit to the frequency of diagnosis. Consequently, a certain proportion of lesions may be unavoidably overlooked. With the popularization of endoscopic submucosal dissection for early gastric cancers and reductions in the extent of resection, remnants of such microcancers are viewed as problematic [4, 5]. However, the occurrence of cancers in the remaining stomach (remnant gastric cancers) can be prevented with reoperation if such patients can be identified during postoperative histopathological examinations. Because remnant gastric cancer was observed in five of the six patients who underwent re-resection, re-resection of the remaining stomach must be performed in principle for patients with marginally positive lesions even if these lesions are microcancers.

Thus, the technique that we describe in this report is an easy and useful method for the efficient identification of lesions extending to the resection margin and prevention of cancers in the remaining stomach (remnant gastric cancers). Further, this technique enables the diagnosis of lesions that may have been overlooked, and thus overcomes the limitations of other methods.

\section{References}

1. Japanese Gastric Cancer Association. Japanese classification of gastric carcinoma, 3rd English edition. Gastric Cancer. 2011;14: 101-12.

2. Kumagai K. Clinicopathological study of multiple early gastric carcinomas and surrounding gastric mucosa. Nihon Geka Gakkai Zasshi. 1982;83:285-95 (in Japanese).

3. Yoshikawa T, Kitamura M, Arai K, Awane Y, Kohsaki G. Clinicopathological study of multiple early gastric cancer. Nihon Shokaki Geka Gakkai Zasshi. 1989;22:1062-6 (in Japanese).

4. Arai K, Kitamura M, Miyashita K. Some aspects of limited operation and endscopic mucosal resection for early gastric cancer. Nihon Shokaki Geka Gakkai Zasshi. 1992;41:1953-7 (in Japanese).

5. Hoya Y, Matai K, Hirabayashi T, Oda Y, Masaoka N, Khono S, et al. Preferred therapeutic choice for synchronous multiple early gastric cancer. Jpn J Surg. 1997;58:280-3 (in Japanese).

6. Kodera Y, Yamamura Y, Torii A, Uesaka K, Hirai T, Yasui K, et al. Incidence, diagnosis and significance of multiple gastric cancer. Br J Surg. 1995;82:1540-3.

7. Huguier M, Ferro L, Barrier A. Early gastric carcinoma: spread and multicentricity. Gastric Cancer. 2002;5:125-9.

8. Moertel CG, Bargen JA, Soule EH. Multiple gastric cancer: review of the literature and study of 42 cases. Gastroenterology. 1957;32:1095-103. 
9. Ishihara S, Nakajima T, Ohta K, Ando T, Yanagisawa A, Katoh Y, et al. Clinicopathologic study on multiple gastric cancer. Jpn J Surg. 1991;12:2821-7 (in Japanese).

10. Tomimatsu S, Ichikura T, Mochizuki H. Problems of multiple lesions in the treatment of gastric cancer. Jpn J Surg. 1997;8: 1723-7 (in Japanese).

11. Kato Y. A pathologist's recommendation to endoscopists regarding microcarcinoma of the Stomach. Shokakinaisikyo. 1991;3:281-90 (in Japanese).

12. Nomiyama Y, Fuchigami T, Yao T, Seo M, Iwashita A. Statistical analysis of the defferences between detected and missed lesions in multiple gastric cancers. Gastroenterol Endosc. 1997; 39:1062-71 (in Japanese).

13. Baba Y, Nakamura K, Sugano H, Takagi K, Kumakura K, Nishi M. Multiple gastric cancer: problem of grossly overlooked synchronous second primary foci near the resection line and their possible evaluation as metachronous second cancer. Jpn J Cancer Clin. 1973;19:912-21 (in Japanese).

14. Folli S, Dente M, Dell'Amore D, Gaudio M, Nanni O, Saragoni L, et al. Early gastric cancer: prognostic factors in 223 patients. Br J Surg. 1995;82:952-6.

15. Sano T, Sasako M, Kinoshita T, Maruyama K, et al. Recurrence of early gastric cancer. Cancer (Phila). 1993;72:3174-8.

16. Ohida M, Uesugi H, Sugano S, Imaizumi H, Tanabe S, Koizumi $\mathrm{W}$, et al. Electoronic endoscopic diagnosis of minute gastric cancer. Stomach Intest. 1995;30:1231-7 (in Japanese).

17. Fuchigami T, Nomiyama Y, Hirakawa M, Matuzaka T, Iwashita M, Nakanishi M, et al. The limit of clinical diagnosis of minute gastric cancer. Stomach Intest. 1995;30:1249-60 (in Japanese). 